\title{
Barriers to increasing fruit and vegetable intakes in older people in Northern Ireland
}

\author{
K. M. Appleton ${ }^{1}$, R. McGill ${ }^{1}$, C. Neville ${ }^{2}$ and J. V. Woodside ${ }^{2}$ \\ ${ }^{1}$ School of Psychology, Queen's University, Belfast, Belfast BT9 5BP, UK and ${ }^{2}$ School of Medicine, \\ Dentistry and Biomedical Sciences, Queen's University, Belfast, Belfast BT12 6BJ, UK
}

The health benefits of a high consumption of fruit and vegetables are now well recognised ${ }^{(1)}$, yet low intakes continue to be reported throughout the $\mathrm{UK}^{(2,3)}$. In attempts to improve fruit and vegetable intakes, various previous researches have identified a number of barriers to increasing fruit and vegetable intakes in the general population ${ }^{(4)}$. The importance of some of these factors, however, is likely to be differ between younger and older adults, and may change over time and location. This project investigated barriers to increasing fruit and vegetable intakes in a large sample of older people in Northern Ireland.

Names and telephone numbers of 1000 people over the age of 65 years living in Northern Ireland were obtained. Attempts were made to contact all individuals by telephone to investigate fruit and vegetable intakes, responses to 22 closed response and one open response question investigating the potential barriers to increasing intakes. Demographic and lifestyle characteristics that have previously been found to affect diet were also assessed.

Data were gained from 426 individuals, representative of the Northern Irish older population according to the 2001 census. These individuals reported consuming a mean of $4.1 \pm 1.3$ portions of fruit and vegetables per $\mathrm{d}$, ranging from $0-8$ portions, with lower intakes in males, older individuals and those living in more deprived areas ${ }^{(3)}$. The principle component analysis of the 22 closed response questions revealed five factors associated with fruit and vegetable intakes - awareness of recommendations, liking, the ease of consumption, difficulties with consumption and willingness to change. Using regression, portions of fruit and vegetables consumed were associated with greater willingness to change $(B=0.197, P<0.01)$, a greater liking of fruit and vegetables $(B=0.675, P<0.01)$ and a greater awareness of recommendations $(B=0.281, P<0.01)$. These relationships remained if demographic and lifestyle characteristics were added to the model, although willingness to change was important only for females. The ease of consumption and difficulties with consumption were not found to be important, and in fact high levels of ease and low levels of difficulties were reported. Similar findings for awareness and liking were also found in those consuming less than five portions of fruit and vegetables per day, in males, older individuals and those living in more deprived areas. Similar patterns were also found in response to the open-ended question.

Associations between fruit and vegetable intakes and an awareness or knowledge of the benefits of fruit and vegetable consumption, a liking for fruits and vegetables and increased willingness to change have all been previously found elsewhere ${ }^{(4,5)}$, and interventions targeting these factors have been found to result in improvements ${ }^{(5,6)}$. These findings suggest that interventions to increase fruit and vegetable intakes in the older population of Northern Ireland should focus on improving knowledge of the benefits and guidelines for fruit and vegetable consumption, improving the awareness of different fruits and vegetables and their products, and strategies that may increase willingness to change. Interventions for the whole population will also be equally applicable to vulnerable groups.

1. World Health Organisation (1990) WHO Technical Report Series 797. Geneva: WHO.

2. Finch, S, Doyle W et al (1998) NDNS: People Aged 65 Years and Over. London: HMSO.

3. Appleton KM, McGill R \& Woodside JV (2009) Br J Nutr 102, 949-953.

4. Cox DN, Anderson AS \& Lean MEJ (1998) Publ Health Nutr 1, 61-68.

5. Gibson EL \& Wardle J (2001) Appetite 37, 91-101.

6. Arnold CG \& Sobal J (2000) J Nutr Educ 32, 130-138. 